\title{
CORRELACIÓN DEL GROSOR ECOGRÁFICO VESICAL CON LOS DATOS CLINICOS Y URODINÁMICOS EN LA HIPERPLASIA BENIGNA DE PRÓSTATA (HBP) SINTOMÁTICA
}

\author{
Jesús Salinas Casado, Santiago Méndez Rubio, F. Campanario Pérez, Miguel Virseda \\ Chamorro, Germán Martínez Urzay, Humberto Pelaquim y Ángel Silmi Moyano.
}

Servicio de Urología. Hospital Clínico de San Carlos. Universidad Complutense de Madrid. Madrid. España.

\begin{abstract}
Resumen.- OBJETIVO: Nuestro objetivo es comprobar la utilidad diagnóstica de la medida del grosor ecográfico de la pared vesical, y establecer la correlación con los hallazgos clínicos y urodinámicos, en pacientes con HBP sintomática.
\end{abstract}

MÉTODOS: Se realizó un estudio transversal prospectivo ("cross-sectional study") en una serie de 74 varones, ledad $\bar{\chi}=72,4 \mathrm{~S}=7,1$ (56-84 años), con HBP sintomática. Los pacientes rellenaron el cuestionario IPSS y se determinó el nivel plasmático de PSAt (ng/ml). Antes de realizar el estudio urodinámico, se determinó mediante ecografía transabdominal (transductor de 3,5 MHz), el volumen prostático (cc) mediante la fórmula del elipsoide, y el grosor de la pared vesical (mm), medido en la

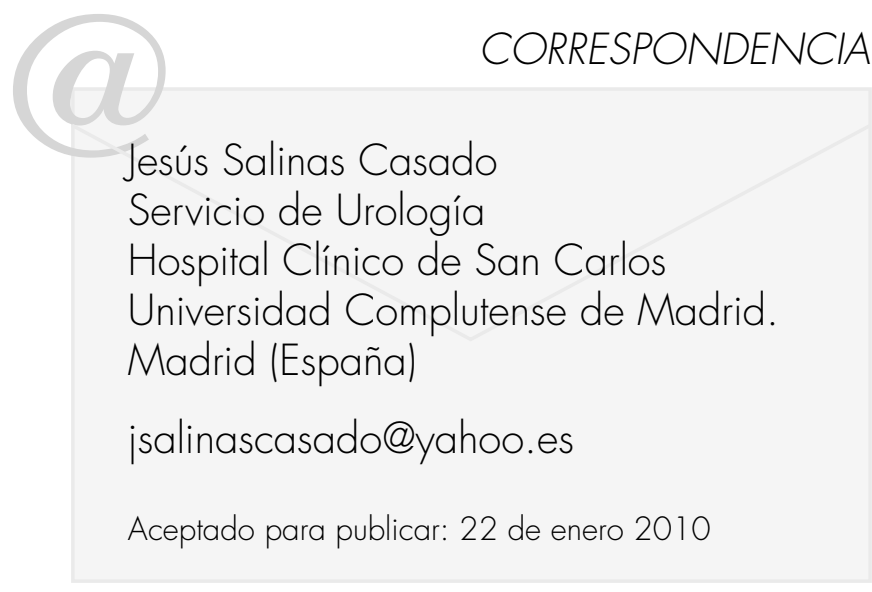

cara anterior. El análisis estadístico se realizó utilizando el ANOVA, el coeficiente de correlación de Pearson, y se construyó una curva de rendimiento diagnóstico (curva ROC).

RESULTADOS: Se demostró una correlación significativa del grosor ecográfico de la pared vesical con el IPSS $(p=0,001 ; r=0,38)$ (parámetro a su vez altamente correlacionado con el volumen prostático y con el PSA $(p=0,01, r=0,62)$, así como con la hiperactividad del detrusor ( $p=0,03, r=0,21$ ), punto de corte de la curva ROC: $3,85 \mathrm{~mm}$. En cambio, no se demostró una correlación significativa entre el grosor ecográfico de la pared vesical y el diagnóstico urodinámico de obstrucción (número de Abrams y Griffths $p=0,223, r=0,14$ ), ni con la potencia contráctil del detrusor, medida en la potencia a flujo máximo (PW) $(p=0,642 r=-0,55)$, ni con la edad ( $p=0,303 ; r=0,12)$. Tampoco se demostró correlación con otras medidas no invasivas urodinámicas (flujo miccional máximo de la flujometría libre $(p=0,318 ; r=0,12)$ y porcentaje del residuo postmiccional $(p=0,696 ; r=0,05)$.

CONCLUSIONES: La medida ecográfica del grosor de la pared vesical, aunque no ha demostrado su utilidad, en nuestra serie, para el diagnóstico de la fase miccional lobstrucción del tracto urinario inferior y afectación contráctil del detrusor), si por el contrario, puede ser introducida como técnica diagnóstica no invasiva de las alteraciones de la fase de llenado, en la forma de hiperactividad del detrusor, en los pacientes con HBP sintomática, presentando además correlación significativa con el crecimiento prostático.

Palabras clave: HBP. Ecografía. Hiperactividad detrusor. Obstrucción tracto urinario inferior. 
Summary.- OBJECTIVES: Our objective is to verify the diagnostic usefulness of ultrasound measurement of the thickness of the bladder wall, and the correlation with clinical/ urodynamic findings in patients with symptomatic $\mathrm{BPH}$.

METHODS: We performed a prospective cross-sectional study (cross-sectional study ") in a series of 74 males lage $\bar{\chi}=72.4 \mathrm{~S}=7.1$ (56-84 years) with symptomatic BPH. Patients completed the IPSS and determined the plasma level of PSAt (ng / mll. Before performing the urodynamic study, was determined by transabdominal ultrasonography (3.5 MHz transducer): prostate volume (cc) by the ellipsoid formula, and bladder wall thickness $(\mathrm{mm})$ measured on theanterior bladder wall. Statistical analysis was performed using ANOVA, the Pearson correlation, and ROC curve.

RESULTS: It was a significant correlation of the sonographic thickness of the bladder wall with the IPSS IP $=0001, r=0.38$ ) (parameter in turn highly correlated with prostate volume and PSA $(p=0.01, r=0.62)$, as well as the detrusor hyperactivity $(p=0.03, r=0.211$, cut off ROC curve: $3.85 \mathrm{~mm}$. Instead, it was not shown a significant correlation between the sonographic thickness of the bladder wall and the urodynamic diagnosis of obstruction (Abrams and number Griffths $p=0223$, $r=0.14$ ) or the detrusor contractile power, measured in power at peak flow $(P w)(p=0642 r=-0.55)$, nor with age $(p=0303, r=0.12)$. Neither correlation was observed with other non-invasive urodynamic measures (the maximum urinary flow flowmetry $(p=0318, r=0.12)$ and percentage postmicturition residual $(p=0696, r=$ 0.0511.

CONCLUSIONS: The ultrasound measurement of bladder wall thickness, although it has not proved useful ness in our series in the diagnosis of the voiding phase llower urinary tract obstruction and detrusor contractility impairment), on the contrary it can be introduced as a diagnostic technique for non-invasive studies in the alterations of the filling phase, in the form of detrusor hyperactivity in also patients with symptomatic $\mathrm{BPH}$, and present a significant correlation with prostate growth.

Keywords: BPH. Ultrasound. Detrusor hyperactivity. Lower urinary tract obstruction.

\section{INTRODUCCIÓN}

La búsqueda de métodos cada vez menos invasivos en los estudios urodinámicos responde no sólo a una mayor comodidad y aceptabilidad por parte del paciente de la prueba, sino, y lo que nos parece más importante, un intento de interferir lo menos posible en la patofisiología del tracto urinario inferior a la hora de su estudio. Se han realizado aproximaciones con métodos urodinámicos no invasivos como resumen en su trabajo Belal M y Abrams $P$ (1) (flujometría libre, catéter condón, manguito, doppler) y con métodos no urodinámicos según los mismos autores (2) (medida ecográfica del grosor vesical o de estimación del peso de la vejiga), aunque aún quedan lejos de poder sustituir al "gold estándar" test presión detrusor/flujo.

La medida del grosor de la pared vesical mediante la ecografía se ha utilizado como test de cribado tanto para la obstrucción del tracto urinario inferior como para la hiperactividad vesical, en hombres y en mujeres, si bien es verdad que con mayor frecuencia en el caso de la obstrucción y en varones. Manieri et al. (3) encontró que la medida del grosor de la pared vesical, aunque no sustituía a la realización del estudio urodinámico, era un predictor útil de la obstrucción del tracto urinario inferior, incluso superior a la flujometría libre.

La hipertrofia del detrusor se ha descrito asociada a numerosas alteraciones del tracto urinario inferior. Generalmente se ha descrito asociada a obstrucción, como resultado de la contracción isométrica del detrusor contra un esfínter cerrado u otra causa de obstrucción. Elbadawi A et al. (4) estudió su ultrestructura, obteniendo datos de cambios, cuando existe obstrucción, como la hipertrofia muscular, con o sin degeneración, alteraciones en el acoplamiento eléctrico (que podría tener un papel en la hiperactividad) y el exceso de fibras elásticas.

Nuestro objetivo es comprobar la utilidad diagnóstica de la medida del grosor ecográfico de la pared vesical, y establecer la correlación con los hallazgos clínicos y urodinámicos, en pacientes con HBP sintomática.

\section{MATERIAL Y MÉTODOS}

Se realizó un estudio transversal prospectivo («cross sectional study») en una serie de 74 varones de edad media 72,4 años (desviación típica: 7,1 años), (intervalo entre 56 y 84 años), remitidos a la Unidad de Urodinámica para su estudio, por presentar hiperplasia benigna prostática sintomática (HBPS).

Los síntomas propios de la Hiperplasia Benigna de Próstata (HBP) se punturaron, mediante el cuestionario autocompletado IPSS (Internacional Prostatic Sintoms Score), traducido y validado al español.

Como determinación analítica, se determinó el nivel plasmático del antígeno prostático específico total (PSAt), en $\mathrm{ng} / \mathrm{ml}$. 
Antes de realizar el estudio urodinámico, se determinó mediante ecografía transabdominal con un transductor de $3,5 \mathrm{MHz}$, el volumen prostático (cc) mediante la fórmula del elipsoide, y el grosor de la pared vesical $(\mathrm{mm})$, medido en la cara anterior.

El estudio urodinámico consistió en una flujometría libre con medida del flujo miccional máximo $(\mathrm{ml} / \mathrm{s})$, volumen miccional (en $\mathrm{ml}$ ) y residuo postmiccional (en proporción respecto al volumen miccional), una cistomanometría con medida de la capacidad cistométrica máxima (en $\mathrm{ml}$ ), y máxima presión del detrusor en esta fase (en $\mathrm{cm} \mathrm{H}_{2} \mathrm{O}$ ) y un estudio presión/flujo con determinación del flujo miccional máximo $(\mathrm{ml} / \mathrm{s})$ y máxima presión del detrusor (en $\mathrm{cm}$ $\mathrm{H}_{2} \mathrm{O}$ ). A partir de estos valores, se determinó el estado funcional del tracto urinario inferior durante esta fase, utilizando el nomograma propuesto por la ICS y Blaivas y de la Rocha (5). También se calculó el número de Abrams \& Griffiths (PQmax - 2Qmax). $\mathrm{Si}$ mayor de $40 \mathrm{~cm} \mathrm{H} \mathrm{H}_{2} \mathrm{O}$ : obstrucción. Si menor de $20 \mathrm{~cm} \mathrm{H}$ O: no obstrucción. Entre 20 y $40 \mathrm{~cm} \mathrm{H} \mathrm{H}_{2} \mathrm{O}$ : indeterminado. Así mismo, se obtuvo la resistencia cuadrática y la potencia a flujo máximo (6).

Se utilizó un equipo de urodinámica UD2000 , catéter urodinámico $7 \mathrm{~F}$ y un catéter rectal $9 F$.

La terminología empleada es la propuesta por la Internacional Continence Society, cuando no se especifique lo contrario.

Se consideró contracción involuntaria del detrusor, mínimamente representativa, a toda elevación fásica de la presión del detrusor, durante el llenado vesical, mayor de $15 \mathrm{~cm} \mathrm{H} \mathrm{H}_{2} \mathrm{O}$ (ICS, 1978) (7) como cifra arbitraria, aceptada por la ICS en 1978. Se optó por ella para evitar posibles artefactos y falsos positivos y simplificar la interpretación de los estudios. De esta manera, tratamos de identificar, de manera fiable, sin ningún género de dudas, a los pacientes con hiperactividad del detrusor. En la actualidad este criterio ha sido sustituido por la nueva terminología de la ICS, definiendo la hiperactividad del detrusor como la observación urodinámica de contracciones involuntarias del detrusor durante la fase de llenado, espontáneas o provocadas (8).

La obstrucción del tracto urinario inferior viene definida por la demostración de una presión máxima del detrusor en el vaciamiento superior a 40 $\mathrm{cm}$ de $\mathrm{H}_{2} \mathrm{O}$, con un flujo miccional máximo menor a $12 \mathrm{ml} / \mathrm{sg}$. Por detrusor acontráctil entendemos la ausencia de contracción voluntaria del detrusor durante el estudio urodinámico. Por insuficiencia de la capacidad contráctil del detrusor, la demostración durante el test de presión detrusor/flujo miccional de una contracción voluntaria máxima de menos de $30 \mathrm{~cm}$ de $\mathrm{H}_{2} \mathrm{O}$, que genera un flujo miccional máximo de menos de $12 \mathrm{ml} / \mathrm{sg}$. Existe un grupo equívoco o indeterminado cuando la presión máxima del detrusor se encuentra entre 30 y $45 \mathrm{~cm} \mathrm{H} \mathrm{H}_{2} \mathrm{O}$ y el flujo miccional máximo entre 12 y $15 \mathrm{ml} / \mathrm{sg}$. Un test normal en el varón sería cuando existe un flujo miccional máximo al menos de $15 \mathrm{ml} / \mathrm{sg}$, con una presión máxima del detrusor inferior a $75 \mathrm{~cm} \mathrm{H} \mathrm{H}_{2}$ (ICS, 1987, 1988; Salinas et al, 1995).

El análisis estadístico se realizó utilizando el análisis de la varianza (ANOVA), para comparar variables categóricas y paramétricas y el coeficiente de correlación de Pearson para comparar variables paramétricas. Para determinar el punto de corte diagnóstico del grosor de la pared vesical relacionado con la presión de llenado se construyó una curva de rendimiento diagnóstico (curva ROC). El nivel de significación (error alfa) se fijó en el $95 \%$ bilateral.

\section{RESULTADOS}

Se demostró una correlación significativa del grosor ecográfico de la pared vesical con el IPSS $(p=0,001 ; r=0,38)$ (parámetro a su vez altamente correlacionado con el volumen prostático y con el PSA $(p=0,01, r=0,62))$, así como con la hiperactividad del detrusor $(p=0,03, r=0,21)$, obteniendo un punto de corte de la curva ROC: $3,85 \mathrm{~mm}$.

En cambio, no se demostró una correlación significativa entre el grosor ecográfico de la pared vesical y el diagnóstico urodinámico de obstrucción (número de Abrams y Griffths $p=0,223, r=0,14$ ) ni con la potencia contráctil del detrusor, medida en la potencia a flujo máximo $(\mathrm{Pw})(\mathrm{p}=0,642 \mathrm{r}=-0,55)$, ni con la edad ( $p=0,303 ; r=0,12)$. Tampoco se demostró correlación con otras medidas no invasivas urodinámicas (flujo miccional máximo de la flujometría libre $(p=0,318 ; r=0,12)$ y porcentaje del residuo postmiccional $(p=0,696 ; r=0,05))$.

\section{Estadística descriptiva}

La distribución de las variables clínicas (IPSS), analíticas (PSA) y ecográficas (tamaño prostático y grosor ecográfico de la pared vesical) se muestran en la Tabla 1.

La distribución de las variables urodinámicas y de los parámetros de la fase miccional se muestran en la Tabla II. Según el monograma de la ICS y Blaivas para valorar la obstrucción, sólo hubo un caso de paciente no obstruido, 49 casos de pacientes con 
TABLA I. DISTRIBUCIÓN DE LAS VARIABLES CLIINICAS ANALITICAS Y ECOGRÁFICAS DEL ESTUDIO.

\begin{tabular}{|l|c|c|}
\hline Variable & Media & Desviación típica \\
\hline Puntuación I-PSS & 12,66 & 3,51 \\
\hline PSA $(\mathrm{ng} / \mathrm{ml})$ & 2,37 & 1,92 \\
\hline Volúmen prostático $\left(\mathrm{cm}^{3}\right)$ & 45,27 & 16,11 \\
\hline Grosor ecográfico pared vesical $(\mathrm{mm})$ & 3,84 & 0,72 \\
\hline
\end{tabular}

obstrucción, 6 casos con insuficiencia contráctil y 18 casos de diagnóstico indeterminado.

\section{Estadística bivariante}

Se observaron correlaciones significativas del grosor ecográfico de la pared vesical con el valor del I-PSS, PSA, volumen prostático y presión máxima del detrusor (en forma de hiperactividad del detrusor) en la cistomanometría.
La mayor correlación del grosor ecográfico de la pared vesical fue con el volumen prostático $(r=$ $0,62, p=0,000$ ) (Figura 1) y con el valor de I-PSS ( $r=$ $0,38, p=0,001$ ) (Figura 2), parámetro a su vez altamente correlacionado con el volumen prostático. Los otros dos parámetros que se relacionaron con el grosor ecográfico de la pared vesical fueron el PSA $(r=0,29$, $p=0,01$ ) (Figura 3) y la presión máxima del detrusor durante la cistomanometría, en la forma de hiperactividad del detrusor $(r=0,25, p=0,03)$ (Figura 4), parámetros que a su vez estaban correlacionados entre sí.

TABLA II. DISTRIBUCIÓN DE LAS VARIABLES URODINÁMICAS DEL ESTUDIO.

\begin{tabular}{|c|c|c|c|}
\hline Variable & & Media & Desviación típica \\
\hline \multirow[t]{3}{*}{ Flujometría libre } & Flujo máximo $(\mathrm{ml} / \mathrm{s})$ & 15,01 & 15,38 \\
\hline & Volumen miccional (ml) & 175,66 & 124,46 \\
\hline & Residuo postmiccional (proporción) & 0,63 & 1,37 \\
\hline \multirow[t]{2}{*}{ Cistomanometría } & Capacidad (ml) & 257,49 & 135,68 \\
\hline & Presión máxima $\left(\mathrm{cm} \mathrm{H}_{2} \mathrm{O}\right)$ & 54,31 & 38,73 \\
\hline \multirow[t]{6}{*}{ Presión flujo } & Flujo máximo $(\mathrm{ml} / \mathrm{s})$ & 9,23 & 4,42 \\
\hline & Presión máxima $\left(\mathrm{cm} \mathrm{H}_{2} \mathrm{O}\right)$ & 75,09 & 34,22 \\
\hline & Residuo postmiccional & 40,97 & 76,15 \\
\hline & № de Abrams \& Griffiths (cm $\left.\mathrm{H}_{2} \mathrm{O}\right)$ & 56,64 & 34,17 \\
\hline & Potencia a flujo máximo (miliwatios) & 71,33 & 48,06 \\
\hline & Resistencia cuadrática $\left[\mathrm{cm} \mathrm{H} \mathrm{H}_{2} \mathrm{O}(\mathrm{ml} / \mathrm{s})^{2}\right]$ & 1,78 & 2,60 \\
\hline
\end{tabular}


No se observó correlación del grosor ecográfico de la pared vesical con ninguna de las variables de la fase miccional (Figura 5). El ANOVA del grosor ecográfico de la vejiga en las diferentes categorías de la clasificación de la ICS-Blaivas tampoco demostró relaciones significativas.

Tomando como normal una presión máxima del detrusor durante la fase de llenado de $20 \mathrm{~cm} \mathrm{H}_{2} \mathrm{O}$ (ausencia de hiperactividad del detrusor), la curva de rendimiento diagnóstico (curva $\mathrm{ROC}$ ) indicó que el punto de corte del grosor ecográfico de la pared vesical fue de $3,85 \mathrm{~mm}$. Con este valor la sensibilidad, para una presión máxima del detrusor durante el llenado (ausencia de hiperactividad del detrusor), es de $58,1 \%$ y la especificidad del $66,7 \%$ (Figura 6 ). Una vez más se tomó este criterio en orden a la simplificación, si bien en la actualidad se define como función del detrusor normal la que permite el llenado vesical con poco o ningún cambio en la presión y además sin contracciones involuntarias fásicas a pesar de la provocación

\section{DISCUSIÓN}

\section{Aspectos metodológicos ecográficos}

Hakenberg et al. (9), mediante ecografía suprapúbica, utilizando un transductor $3,5 \mathrm{MHz}$, describen el grosor de la pared vesical anterior en $3 \pm$

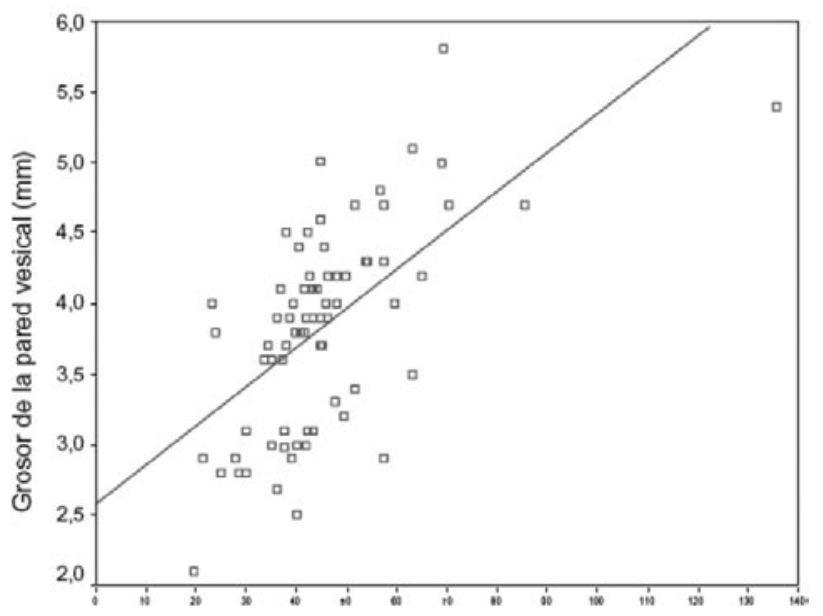

Volumen prostático (cm 3)

FIGURA 1. Recta de regresión del grosor ecográfico vesical respecto al volumen prostático.

Se demostró una correlación significativa del grosor ecográfico de la pared vesical con el volumen ecográfico prostático $(r=0,62, p=0,00)$.
$1 \mathrm{~mm}$, en mujeres sanas (edad media: 37,8 años) y $3,3 \pm 1,1 \mathrm{~mm}$ en varones sanos (edad media: 39,9 años) (Figura 7). Esto es, el grosor de la pared vesical tiende a ser un poco mayor en el varón, así como se comprueba un pequeño aumento del grosor vesical, con la edad, en ambos géneros. Por otra parte, se demostró una pequeña disminución del grosor ecográfico de la pared vesical con el incremento del volumen intravesical, aunque desde un punto de vista práctico, podría considerarse despreciable.

Oelke et al. (10) describen el grosor ecográfico de la pared anterior del detrusor utilizando un transductor de 7,5 MHz suprapúbico. La adventicia y mucosa vesical aparecen hiperecogénicas, mientras que el detrusor aparece hipoecogénico. La distancia entre las dos líneas hiperecogénicas representa el grosor del detrusor.

El grosor ecográfico de la pared vesical (Hakenberg et al 2000) sería más alto que el del detrusor (Oelke et al 2006), ya que añaden el espesor de la adventicia y la mucosa al del detrusor. Por otra parte, en ocasiones, el tejido perivesical no puede diferenciarse de la adventicia, ya que ambas son hiperecogénicas $y$, sobre todo, cuando se utiliza un transductor de $3,5 \mathrm{MHz}$. Tampoco en este caso encuentran que la pequeña disminución del espesor con el incremento de llenado vesical parece que tenga importancia práctica.

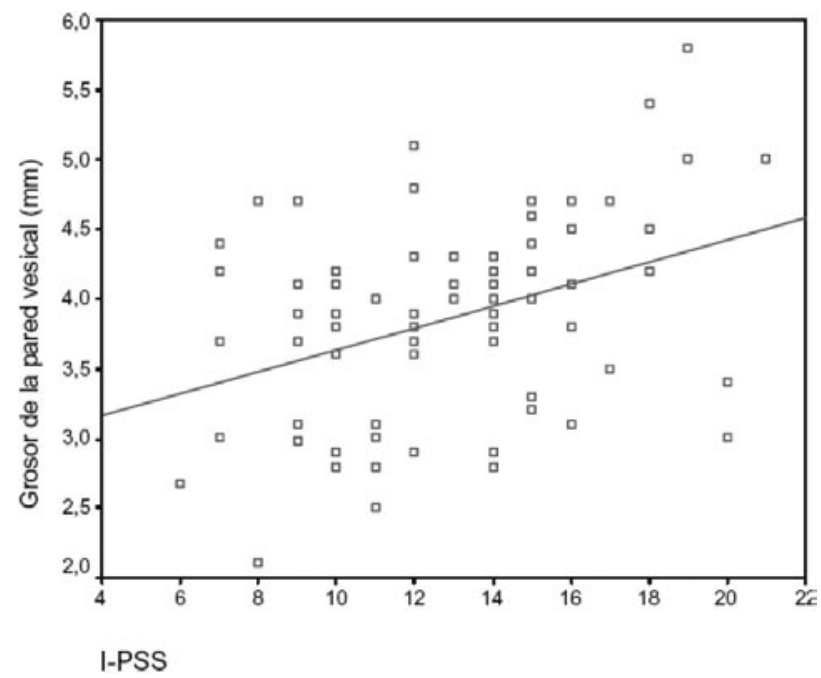

FIGURA 2. Recta de regresión del grosor ecográfico vesical respecto a la puntuación IPSS.

Se demostró una correlación significativa del grosor ecográfico de la pared vesical con el valor del I-PSS $(r=0,38, p=0,001)$, parámetro, a su vez, altamente correlacionado con el volumen prostático. 
El grosor ecográfico del detrusor de la serie de Oelke et al (2006) correspondió a 55 adultos normales, de 15 a 40 años de edad, de ambos sexos, y con un llenado vesical completo. En 9 se midió hasta un volumen de $300 \mathrm{ml}$, con incrementos de $50 \mathrm{ml}$ y de $100 \mathrm{ml}$ hasta el llenado completo. Los valores significativos para el varón fueron de 1,4 mm, frente a 1,2 $\mathrm{mm}$ en mujeres. La edad y el índice de masa corporal no presentaron relación significativa.

El grosor ecográfico del detrusor de esta serie disminuyó rápidamente durante los primeros 250 $\mathrm{ml}$ de llenado (o el 40-50\% de la capacidad vesical). Posteriormente, hasta alcanzar la máxima capacidad vesical, no mostró diferencias significativas. En este sentido, este último dato contrastaría con el descrito por Hakenberg et al (2000), que no comprueban una diferencia significativa del grosor ecográfico de la pared vesical con el volumen intravesical.

Algunos autores (11) aconsejan, desde un punto de vista metodológico, utilizar un aumento de 9,8 veces, de la imagen ecográfica de la pantalla, en orden a obtener una mayor exactitud de la medida del grosor ecográfico del detrusor.

\section{Edad}

No se ha detectado en nuestro estudio correlación significativa del grosor ecográfico de la

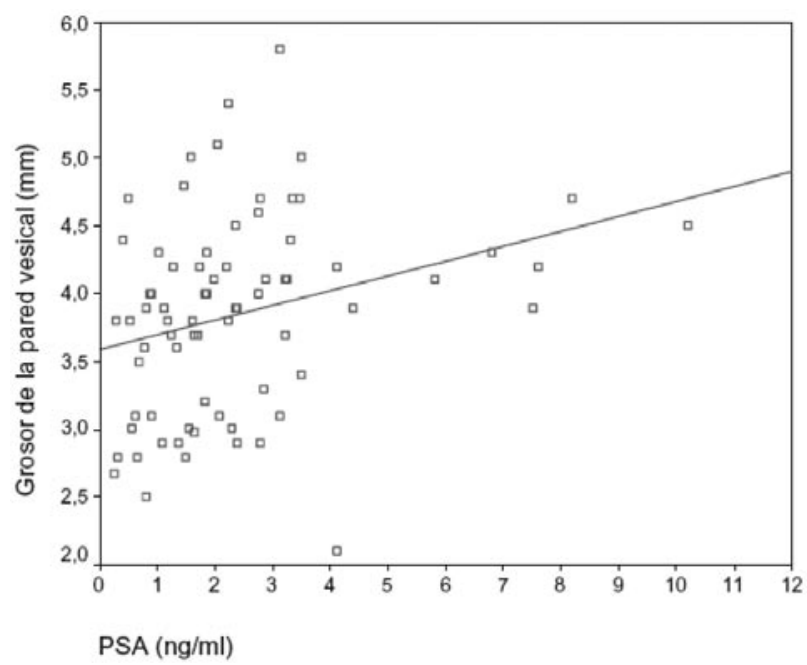

FIGURA 3. Recta de regresión del grosor ecográfico vesical respecto al valor del PSA.

Se demostró una correlación significativa del grosor ecográfico de la pared vesical con el PSA / $r=0,29$, $p=0,011$, que a su vez estaba correlacionado con la presión máxima del detrusor durante la cistomanometría. pared vesical con la edad $(r=0,12, p=0,303)$, en semejanza a los datos descritos por Oelke et al (2006). No obstante, en este último trabajo, los pacientes estaban comprendidos entre 15 y 40 años, lo cual contrastó con nuestra serie, cuya edad oscilaba entre 56 y 84 años (media: 72,4 años). En la serie de Hakenberg et al (2000), la edad media de la serie correspondió a 66,4 años y observaron un ligero incremento del grosor vesical ecográfico con la edad $(r=0,12)$.

No obstante, este pequeño incremento no precisaría de una corrección de la medida ecográfica en relación con la edad. Esto contrastaría con los cambios estructurales de la pared vesical descritos con el envejecimiento: hipertrofia muscular, incremento tejido colágeno..., que se asociaron con un aumento de grosor de la pared vesical $(12,13)$. Es de señalar que estos datos pueden corresponder así mismo a la obstrucción del tracto urinario inferior (Gilpin et al 1985).

\section{Síntomas funcionales del tracto urinario inferior}

En nuestro estudio se demostró una correlación del grosor ecográfico de la pared vesical con el valor de I-PSS $(12,66 \pm 3,51)(p=0,001)(r=$ $0,38)$, que cuantifica los síntomas funcionales del tracto urinario inferior (LUTS), de almacenamiento y vaciamiento conjuntamente. Dicho parámetro estaría

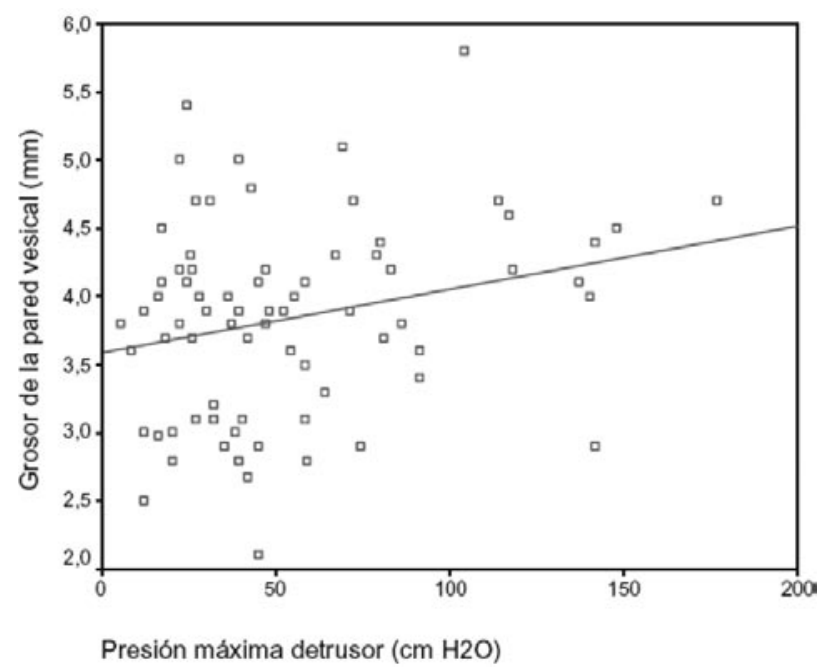

FIGURA 4. Recta de regresión del grosor ecográfico vesical respecto a la presión máxima del detrusor en la cistomanometría.

Se demostró una correlación significativa del grosor ecográfico de la pared vesical con presión máxima del detrusor durante la cistomanometría (hiperactividad del detrusor) ( $r=0,25, p=0,03)$. 
altamente correlacionado, a su vez, con el volumen prostático.

El valor de I-PSS de nuestra serie corresponde al grado moderado. Un I-PSS mayor de 5 fue la cuantificación de los síntomas funcionales del tracto urinario inferior en el trabajo de Hakenberg et al (2000).

Aunque en nuestro trabajo se encontró relación entre LUTS y volumen prostático, no ha sido así en la asociación entre LUTS y obstrucción del tracto urinario inferior.

Un estudio multicéntrico de la ICS-hiperplasia benigna de próstata (15) en 1.271 varones, entre 45 y 88 años, demostró una pobre correlación en este sentido. Este estudio exploró a 933 pacientes con test presión detrusor/flujo miccional, utilizando el cuestionario de ICS varones, para comprobar los síntomas de almacenamiento y vaciamiento. Los datos del estudio no variaron aunque se examinaron por separado los síntomas de almacenamiento y vaciamiento. Sin embargo, otros autores encuentran una correlación entre la obstrucción del tracto urinario inferior y los síntomas de vaciamiento o miccionales $(16,17)$. Es posible que la separación de nuestro cuestionario 1 PSS en síntomas de almacenamiento y vaciamiento o miccionales, nos hubieran sido de más utilidad y pudieron habernos demostrado, así mismo, un ma-

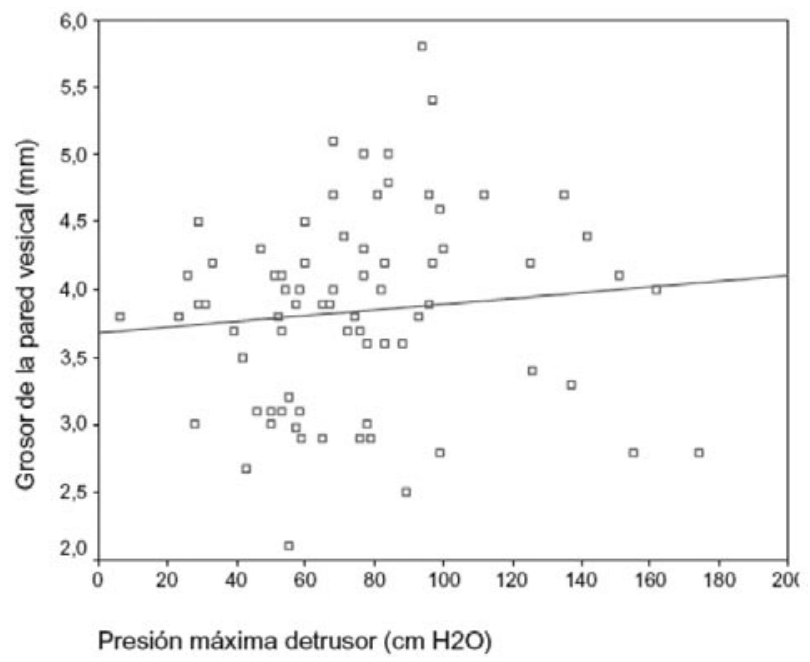

FIGURA 5. Recta de regresión del grosor ecográfico vesical respecto a la presión máxima del detrusor en el estudio presión/flujo.

No se demostró en nuestro estudio correlación significativa entre el grosor ecográfico de la pared vesical y el diagnóstico urodinámico de obstrucción del tracto urinario inferior. yor porcentaje de síntomas de almacenamiento en relación con el diagnóstico urodinámico de hiperactividad del detrusor.

\section{PSA}

En nuestro estudio se demostró una correlación positiva entre el grosor de la pared vesical y PSA $(2,37 \pm 1,92)(p=0,012)(r=0,29)$.

En un estudio de más de 300 pacientes con síntomas del tracto urinario inferior, la relación entre PSA y diagnóstico urodinámico de obstrucción fue valorada y estratificada, utilizando un análisis de regresión logística (18). Si el PSA estaba entre 4 y $6 \mathrm{ng} / \mathrm{ml} \mathrm{la}$ posibilidad de obstrucción del tracto urinario inferior fue del $65 \%$, frente al $81 \%$ si el valor del PSA se encontraba entre 6 y $10 \mathrm{ng} / \mathrm{ml}$. Esta aproximación estaría limitada desde que sólo el $23 \%$ de la población estudiada podría ser estratificada de esta manera y tuvieran un PSA mayor de $4 \mathrm{ng} / \mathrm{ml}$. Por otra parte, esta aproximación se complica aún más, ante la necesidad de descartar un carcinoma de próstata en PSA mayor de $4 \mathrm{ng} / \mathrm{ml}$.

En un estudio más reciente llevado a cabo por Bantis $A$ et al. (19), en el que se estudian 120 pacientes con síntomas del tracto urinario inferior encuentran resultados similares respecto al psa. Hubo

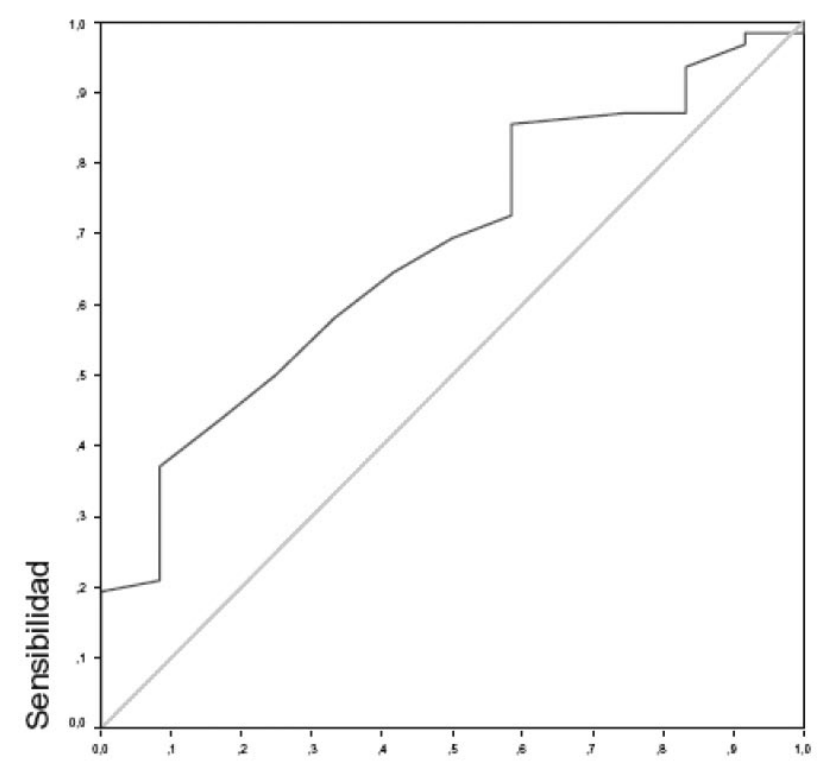

1 - Especificidad

FIGURA 6. Curva de rendimiento diagnóstico del grosor ecográfico vesical frente a la presión máxima del detrusor en la cistomanometría. 
una correlación estadística entre la obstrucción del tracto de salida, el PSA $(p=0,004)$, el volumen prostático con $\mathrm{P}$ de $<0,001$ ) y la protusión intravesical prostática (dato ecográfico) $p=0,005$. Por el contrario, no hay correlación estadística entre el IPSS y la obstrucción $(p=0,228)$, ni con el Qmáx $(p=0,745)$.

\section{Volumen prostático}

En nuestro trabajo se demostró una correlación positiva del grosor ecográfico de la pared vesical y el volumen prostático ecográfico $(45,27 \pm$ $16,11)(p=0,000)(r=0,62)$. También Hakenberg et al (2000) demostraron un moderado aumento del grosor ecográfico vesical $(3,67 \pm 0,11 \mathrm{~mm})$ en hombres con LUTS y crecimiento prostático.

La relación entre el volumen prostático ecográfico total y la obstrucción del tracto urinario inferior ha sido estudiada en diversos trabajos. Un estudio retrospectivo en 521 pacientes demostró una débil, pero significativa correlación estadística, entre el tamaño prostático y la obstrucción del tracto urinario inferior (20). De los pacientes con volumen prostático de más de $40 \mathrm{ml}$, el 70\% tendrían el diagnóstico de obstrucción del tracto urinario inferior. Este grupo presenta el $45 \%$ de la población y esto no es suficiente para diagnosticar la obstrucción del tracto urinario inferior en la mayoría de los pacientes. Estos datos presentaban para un volumen prostático mayor de $40 \mathrm{ml}$, una sensibilidad del $49 \%$ y una especificidad del $32 \%$.

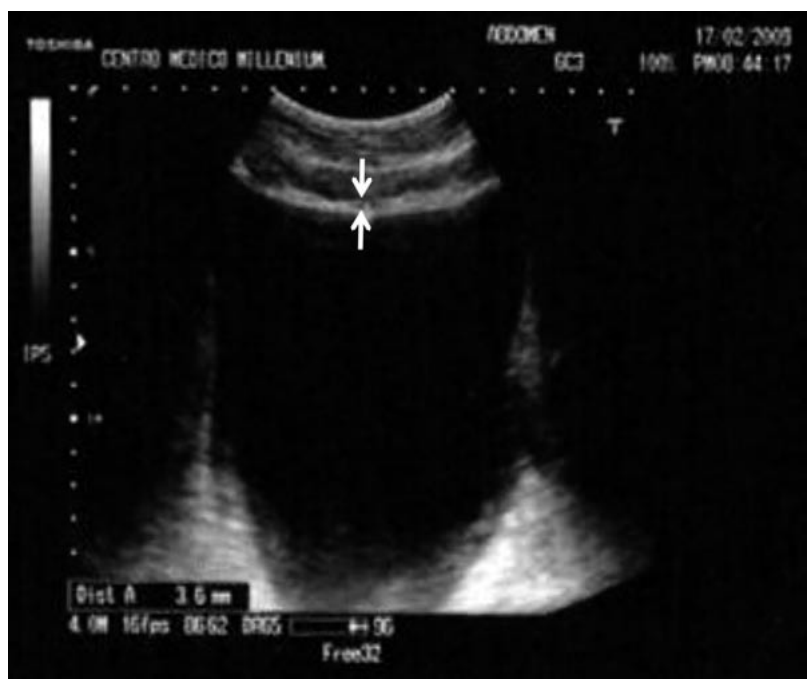

FIGURA 7. Medida del grosor ecográfico de la pared anterior vesical.
En una serie de 525 pacientes, Eckhardt et al. (21) describieron una correlación estadísticamente significativa entre el volumen prostático y la obstrucción del tracto urinario inferior $(p<0,001, r=$ 0,28 ). Sin embargo, inesperadamente, el volumen prostático disminuyó ligeramente en los grados de obstrucción de Schafer altos (grados 5 y 6).

Ante la insuficiencia de valor del volumen ecográfico prostático en el diagnóstico de obstrucción del tracto urinario inferior, se han propuesto otros datos ecográficos como la forma prostática (PCAR), protusión intravesical prostática (IPP) $(22,23)$, proporciones relativas de las diferentes zonas prostáticas e índices (24). Reiss LO et al. (25) obtiene como punto de corte de la medida de la protusión intravesical prostática para indicar obstrucción del TUI de $5 \mathrm{~mm}$, con un $95 \%$ de sensibilidad $(75,1-99,2)$ y el $50 \%$ de especificidad $(28,2-71,8)$.

\section{Obstrucción tracto urinario inferior}

El grosor de la pared vesical lo del detrusor) y el peso vesical son dos de los parámetros más estudiados ecográficamente para el diagnóstico no invasivo de obstrucción del tracto urinario inferior.

El valor del grosor de la pared vesical lo del detrusor), como dato diagnóstico de la obstrucción del tracto urinario inferior se basa en que la obstrucción prostática se asocia a hipertrofia del detrusor, como se ha podido demostrar mediante estudios estructurales (26).

El grosor ecográfico de la pared vesical está influenciado por el volumen vesical, de tal forma, que la pared vesical se adelgaza conforme el volumen intravesical se incrementa.

Para superar este factor, diversos autores han propuesto distintas soluciones, como la realización de índices complejos (27), o utilizar constantes volúmenes intravesicales de $150 \mathrm{ml}$ (Manieri et al 1998), aunque otros demuestran que a partir de $250 \mathrm{ml}$ de volumen intravesical, las medidas no varían (Oelke et al 2006).

Manieri et al (1998) superaron el problema del volumen vesical midiendo ecográficamente el grosor de la pared vesical a un constante valor de $150 \mathrm{ml}$ durante el estudio urodinámico en adultos. Un total de 174 varones con síntomas funcionales del tracto urinarior inferior (LUTS) fueron estudiados con tres medidas ecográficas de grosor en tres puntos: anterior y paredes laterales. El diagnóstico de obstrucción urodinámica se realizó mediante el número 
de Abrams-Griffiths. De los 58 pacientes con un grosor ecográfico de la pared abdominal mayor de 5 $\mathrm{mm}$, el $88 \%$ presentaban obstrucción en los estudios presión/flujo, con una especificidad del $92 \%$, aunque la sensibilidad fue del $54 \%$.

Otros autores (28) obtienen distintos valores de obstrucción del tracto urinario inferior: 2 $\mathrm{mm}$, con un valor predictivo positivo del $95.5 \%$ para un grosor igual o mayor a $2 \mathrm{~mm}$ una especificidad del $97,3 \%$ y una sensibilidad del $63,6 \%$. No obstante, hay que destacar que estos autores no miden ecográficamente todo el grosor de la pared vesical, sino se limitan al detrusor, con lo que los valores serían, lógicamente, más bajos. Además, estos autores no utilizan como método diagnóstico urodinámico de obstrucción el número de AbramsGriffiths, sino el de Schäfer o clasificación en ajedrez.

Oelke et al (2007) demostraron un diagnóstico no invasivo más exacto de obstrucción del tracto urinario inferior mediante la medida del grosor ecográfico del detrusor, frente a otros métodos no invasivos, como la flujometría, medidas ecográficas del residuo postmiccional y volumen prostático.

Kessler et al. (29), estudiando pacientes con síntomas del tracto urinario inferior sin cirugías prostáticas previas ni patología neurourológica, midiendo el grosor ecográfico del detrusor, utilizando el número de Abrams-Griffiths como parámetro de obstrucción urodinámico, llegan a valores de un grosor mayor de 2,9 mm como dato de alto valor diagnóstico de obstrucción del tracto urinario inferior.

En nuestra serie, a semejanza de la clasificación de Blaivas y Groutz (30), en la obstrucción en la mujer, se utilizó el parámetro de presión máxima miccional del detrusor, junto al flujo miccional máximo, como diagnóstico urodinámico de obstrucción: clasificación clásica ICS-Blaivas (Blaivas y Rocha 1986), no demostrándose relación significativa entre el grosor ecográfico de la pared vesical y la existencia de una obstrucción urodinámica del tracto urinarior inferior. A la misma conclusión llegan otros autores, como Laniado et al. (31).

Una primera explicación para esta discordancia podría estar en la elección del método urodinámico diagnóstico de obstrucción del tracto urinario inferior de estos pacientes.

En nuestra serie se ha utilizado la recomendada por la ICS (Internacional Continence Society 1976 (32) y Blaivas y de la Rocha), por su sencillez de aplicación.
Tampoco la aplicación del estudio de la resistencia cuadrática al diagnóstico de obstrucción del tracto urinario inferior, en nuestra serie ha demostrado relación significativa con la determinación ecográfica del grosor de la pared vesical. Se considera que un coeficiente mayor de 0,25 sugiere obstrucción y si es superior a 0,5 indica obstrucción severa (33).

La potencia contráctil del detrusor ha sido estudiada en nuestra serie, no demostrando relación significativa con el grosor ecográfico vesical. Se expresa en unidades de potencia (miliWatios). Schaefer considera que una potencia superior o igual a 50 $\mathrm{mWatios}$ es normal.

En nuestra serie, a los datos aportados por la presión/flujo se asociaron los valores no invasivos del flujo miccional máximo (de la flujometría libre) y el residuo postmiccional.

Tampoco estos datos mostraron relación significativa con el grosor ecográfico vesical, aunque se pudieran obtener de una manera no invasiva. La flujometría libre no requiere instrumentación alguna al paciente, y el residuo postmiccional se podría obtener por ecografía no invasiva (34).

Aunque el flujo miccional máximo y residuo postmiccional puede estar en relación con la obstrucción del tracto urinario inferior (35), también (y más frecuentemente) está asociado a una hipoactividad del detrusor durante la micción $(36,37)$, en un estudio realizado con pacientes con HBP no tratados, encontró únicamente una disminución en la contractilidad del detrusor y un aumento en la prevalencia de hiperactividad del detrusor.

Sin embargo, Abrams y Griffiths (38) demostraron una utilidad cuando asocian la medición del residuo postmiccional con los valores de la flujometría.

Incluso se ha comparado la medida convencional del espesor de la pared vesical con un dispositivo sonográfico nuevo (BVM 6500), aunque presenta inconvenientes con espesores mayores de $4 \mathrm{~mm}$ y no hay diferencias significativas con las medidas convencionales (39). Estos nuevos dispositivos de escaneo ultrasónico tridimensionales, algunos portátiles, están siendo evaluados.

Otro método ecográfico de valoración de la hipertrofia del detrusor, alternativo a la medición ecográfica del grosor vesical, sería la estimación del peso vesical (40). Este autor, Kojima et al. (41) considera a la vejiga como una esfera o elipsoide y en una serie de 65 pacientes demostró una correlación 
significativa con los parámetros urodinámicos de obstrucción, utilizando tanto el número de AbramsGriffiths, como el PURR del método de Schaefer. Con un peso de 35 gramos, el diagnóstico de obstrucción tendría una sensibilidad del $85 \%$ y una especificidad del $87 \%$.

Aunque la medida ecográfica del peso vesical puede hacerse por vía transabdominal $(42,43)$, algunos autores la realizan por vía transrectal (44). Ukimura $O$ et al (2003) describen un método de estimación del peso de la vejiga a partir del grosor vesical medido ecográficamente y el volumen vesical (45).

Un incremento del grosor ecográfico vesical no siempre corresponde a obstrucción del tracto urinario inferior, sino que ha sido encontrado en el envejecimiento, asociado a hipertrofia del detrusor, fibrosis y pérdida de inervación autonómica (46).

En el mismo sentido, puede ocurrir con el incremento del peso ecográfico vesical, que no siempre corresponde a obstrucción del tracto urinario inferior. El incremento del peso ecográfico vesical define hipertrofia del detrusor, pero no su causa. El peso vesical ecográfico puede estar incrementado en casos de hiperactividad del detrusor, sin obstrucción del tracto urinario inferior (Kojima et al 1997).

\section{Hiperactividad detrusor}

En nuestra serie, se ha demostrado una relación significativa entre el grosor ecográfico vesical y la presión máxima del detrusor durante la fase de llenado vesical, en la forma de hiperactividad del detrusor, tomando como referencia de contracción involuntaria del detrusor toda elevación fásica de la presión del detrusor durante el llenado vesical mayor de $15 \mathrm{~cm} \mathrm{H}_{2} \mathrm{O}$ (47).

Como ya señalamos anteriormente, seguimos este criterio por una cuestión eminentemente práctica, eliminando de esta manera la posibilidad de artefactos en los registros, o la variabilidad en la interpretación en las contracciones no inhibidas de baja amplitud, identificando de manera inequívoca a los pacientes que presentan hiperactividad del detrusor. En la actualidad la ICS define la hiperactividad del detrusor como la observación urodinámica de contracciones involuntarias del detrusor durante la fase de llenado espontáneas o provocadas, sin límite inferior de la amplitud de las contracciones no inhibidas, pero confiando la interpretación de las contracciones de menor amplitud (menores de $5 \mathrm{cmH}_{2} \mathrm{O}$ ) a una técnica urodinámica de alta calidad. (Abrams 2003).
No obstante, algunos autores prefieren considerar también contracciones involuntarias del detrusor cuando superan los $10 \mathrm{~cm} \mathrm{H} \mathrm{H}_{2} \mathrm{O}$ de presión máxima (48), mientras otros consideran como tal, cualquier amplitud de la contracción involuntaria, en el intento de inhibir las mismas (ICS 1976) y hay quien considera hiperactividad del detrusor, ante cualquier elevación fásica de la presión de llenado (49), que se asocia a urgencia miccional.

La presencia de hiperactividad detrusor en pacientes con HBP obstructiva es muy frecuente, alrededor del 50-60\% (50). En una serie de 100 casos con síntomas funcionales del tracto urinario inferior, Salinas et al. (51) demostraron hiperctividad detrusor en el $33 \%$ de los casos, correspondiendo todos ellos a pacientes con obstrucción del tracto urinario inferior. Aproximadamente en el $50-75 \%$ de los pacientes con HBP obstructiva y detrusor hiperactivo, éste desaparece tras la cirugía prostática. Sin embargo, en un pequeño porcentaje persiste la hiperactividad tras la intervención $(52,53)$.

Hyman $M$ et al. (54), en una serie de 160 pacientes varones sin patología neurológica, el síntoma urgencia miccional está altamente correlacionado con el hallazgo de hiperactividad vesical y se encontró asociado a obstrucción en un $46 \%$ de los pacientes con síntomas del tracto urinario inferior.

El tipo de hiperactividad del detrusor no asociado a obstrucción urinaria (idiopática) es más común en la población anciana y ha sido relacionado con cambios degenerativos en la invervación vesical, sin llegar a constituir una afectación neurológica evidente (55).

Otro parámetro urodinámico de interés durante la fase de llenado vesical es la acomodación vesical. La acomodación vesical se expresa en unidades de presión partido por unidades de volumen $1 \mathrm{~cm}$ $\mathrm{H}_{2} \mathrm{O} / \mathrm{ml}$ ). La disminución de la acomodación vesical es debida a una alteración de las propiedades viscoelásticas vesicales. Esta alteración puede ser secundaria a un aumento de la proporción de colágeno de la pared vesical (Susset 1983) o a un aumento del «tono» vesical (56). Para diferenciar ambos procesos habrá que recurrir a la llamada cistomanometría de llenado rápido (Susset 1983).

La disminución de la acomodación vesical produce un aumento de la presión vesical de llenado. Si este aumento sobrepasa los $30 \mathrm{~cm} \mathrm{H} \mathrm{H}_{2} \mathrm{O}$ se produce afectación del tracto urinario superior (57).

No siempre es fácil diferenciar, dentro de una elevación de la presión de llenado vesical, una 
acomodación vesical disminuida, de una hiperactividad del detrusor. Algunos casos de acomodación vesical disminuida en la cistomanometría convencional han correspondido a hiperactividad del detrusor en la urodinámica ambulatoria o telecistomanometría (58).

\section{CONCLUSIONES}

Concluyendo, de los datos de nuestra serie parece que la fase de llenado tiene mayor influencia sobre el grosor de la pared vesical, que la fase de vaciamiento o miccional; lo que podría estar en relación, entre otros, con el factor tiempo (la fase miccional tiene una menor duración que la fase de llenado vesical). Existen una serie de trabajos, aunque referidos a mujeres y niños, que confirman nuestros hallazgos de incremento del grosor ecográfico vesical en la hiperactividad del detrusor (59-62).

En conclusión, la medida ecográfica del grosor de la pared vesical, aunque no ha demostrado su utilidad, en nuestra serie, para el diagnóstico de la fase miccional (obstrucción del tracto urinario inferior y afectación contráctil del detrusor), sí por el contrario, puede ser introducida como técnica diagnóstica de las alteraciones de la fase de llenado no invasiva, en la forma de hiperactividad del detrusor, en los pacientes con HBP sintomática, presentando además correlación significativa con el crecimiento prostático.

\section{BIBLIOGRAFIA y LECTURAS RECOMENDADAS (*lectura de interés $y^{* *}$ lectura fundamental)}

**1. Belal M, Abrams P Noninvasive methods of diagnosing bladder outlet obstruction in men. Part 2: Noninvasive urodynamics and combination of measures. J Urol, 2006; 176(1):29-35. Comment in: J Urol. 2007; 177(2):797; author reply 797-8.

**2. Belal M, Abrams P. Noninvasive methods of diagnosing bladder outlet obstruction in men. Part 1: Nonurodynamic approach. J Urol, 2006; 176(1):22-8.

*3. Manieri C, Carter SS, Romano G, Trucchi A, Valenti M, Tubaro A. The diagnosis of bladder outlet obstruction in men by ultrasound measurement of bladder wall thickness. J Urol. 1998; 159(3):7615.

4. Elbadawi A, Yalla SV, Resnick NM. Structural basis of geriatric voiding dysfunction. IV. Bladder outlet obstruction. J Urol, 1993; 150(5 Pt 2):168195.
5. Blaivas JG, de la Rocha RE. Impaired urinary flow rate: the distinction between bladder outlet obstruction and poor detrusor contractility. AUA Congress. Atlanta, 1986.

**6. Salinas J, Vírseda M, Teba F. Urodinámica de la hiperplasia prostática benigna. In Salinas J, Vírseda M, Teba F, eds. Aplicaciones diagnósticas, pronósticas y terapéuticas. Madrid: Pub Santher, 2000.

7. ICS. Third report on the standardisation of terminology of lower urinary tract function. Procedures related to the evaluation of micturition: pressureflow relationships. Residual urine. Scand J Urol Nephrol, 1978; 12(3):191-3.

8. Abrams P, Cardozo L, Fall M, Griffiths D, Rosier P, Ulmsten U, Van Kerrebroeck P, Victor A, Wein A; Standardisation Sub-Committee of the International Continence Society. The standardisation of terminology in lower urinary tract function: report from the standardisation sub-committee of the International Continence Society. Urol, 2003; 61(1):37-49.

**9. Hakenberg OW, Linne C, Manseck A, Wirth MP.Bladder wall thickness in normal adults and men with mild lower urinary tract symptoms and benign prostatic enlargement. Neurourol Urodyn 2000;19(5):585-93.

**10. Oelke M, Hofner K, Jonas U, Ubbink D, de la Rosette J, Wijkstra H. Ultrasound measurement of detrusor wall thickness in healthy adults. Neurourol Urodyn 2006; 25(4):308-17; discussion 318.

**11. Oelke M, Höfner K, Jonas U, de la Rosette JJ, Ubbink DT, Wijkstra H. Diagnostic accuracy of noninvasive test to evaluate bladder outlet obstruction in men: detrusor wall thickness, uroflowmetry, postvoid residual urine, and prostate volume. Eur Urol 2007;52:827-35.

12. Susset JG, Servot-Viguier D, Lamy F, Madernas P, Black R. Collagen in 155 human bladders. Invest Urol 1978;16(3):204-6.

13. Gilpin SA, Gilpin CJ, Dixon JS, Gosling JA, Kirby RS. The effect of age on the autonomic innervation of the urinary bladder. Br $\mathrm{J}$ Urol 1986;58(4):378-81.

14. Gilpin SA, Gosling JA, Barnard RJ. Morphological and morphometric studies of the human obstructed, trabeculated urinary bladder. Br J Urol 1985;57(5):525-9.

15. De la Rosette JJ, Witjes WP, Schafer W, Abrams P, Donovan JL, Peters TJ, et al. Relationships between lower urinary tract symptoms and bladder outlet obstruction: results from the ICS-«BPH» study. Neurourol Urodyn 1998;17(2):99-108.

16. Neal DE, Styles RA, Powell PH, Ramsden PD. Relationship between detrusor function and residual urine in men undergoing prostatectomy. $\mathrm{Br} \mathrm{J}$ Urol, 1987;60(6):560-6. 
17. Reynard JM, Abrams P. Bladder-outlet obstruction-assessment of symptoms. World J Urol 1995; 13(1):3-8.

18. Laniado ME, Ockrim JL, Marronaro A, Tubaro A, Carter SS. Serum prostate-specific antigen to predict the presence of bladder outlet obstruction in men with urinary symptoms. BJU Int 2004; 94(9):1283-6.

19. Bantis A, Zissimopoulos A, Kalaytzis C, Giannakopoulos S, Soundoulidis P, Agelonidou E, Voudalikakis C, Touloupidis S. [Correlation of serum prostate specific antigen, the volume and the intravesical prostatic protrusion for diagnosing bladder outlet obstruction in patients with benign prostate hyperplasia][Article in Greek, Modern] Hell J Nucl Med. 2007 May-Aug;10(2):138-43.

20. Rosier PF, de la Rosette JJ. Is there a correlation between prostate size and bladder-outlet obstruction? World J Urol 1995b;13(1):9-13.

21. Eckhardt MD, van Venrooij GE, Boon TA. Interactions between prostate volume, filling cystometric estimated parameters, and data from pressure-flow studies in 565 men with lower urinary tract symptoms suggestive of benign prostatic hyperplasia. Neurourol Urodyn 2001;20(5):579-90.

22. Chia SJ, Heng CT, Chan SP, Foo KT. Correlation of intravesical prostatic protrusion with bladder outlet obstruction. BJU Int 2003;91(4):371-4.

23. Ho HS, Lim KB, Foo KT. The importance of intravesical prostate protrusion in predicting bladder outlet obstruction in patients with good urinay flow. Eur Urol Suppl 2007;6(2):68.

24. Witjes WP, Aarnink RG, Ezz-el-Din K, Wijkstra H, Debruyne EM, de la Rosette JJ. The correlation between prostate volume, transition zone volume, transition zone index and clinical and urodynamic investiga-tions in patients with lower urinary tract symptoms. Br J Urol 1997; 80(1):84-90.

25. Reis LO, Barreiro GC, Baracat J, Prudente A, D'Ancona CA. Intravesical protrusion of the prostate as a predictive method of bladder outlet obstruction Int Braz J Urol. 2008 Sep-Oct;34(5):62733; discussion 634-7.

26. Elbadawi A, Yalla SV, Resnick NM. Structural basis of geriatric voiding dysfunction. J Urol 1993;150:1650-5, 1656-67, 1668-80, 1681-95.

27. Kaefer M, Barnewolt C, Retik AB, Peters CA. The sonographic diagnosis of infravesical obstruction in children: evaluation of bladder wall thickness indexed to bladder filling. J Urol 1997;157(3):98991.

*28. Oelke M, Höfner K, Wiese B, Grünewald V, Jonas $\mathrm{U}$. Increase in detrusor wall thickness indicates bladder outlet obstruction (BOO) in men. World J Urol. 2002 Apr;19(6):443-52.

**29. Kessler TM, Gerber R, Burkhard FC, Studer UE, Danuser H. Ultrasound assessment of detrusor thickness in men-can it predict bladder outlet obstruction and replace pressure flow study? J Urol 2006;175(6):2170-3.

30. Blaivas JG, Groutz A. Bladder outlet obstruction nomogram for women with lower urinary tract symptomatology. Neurourol Urodyn 2000;19(5):553-64.

*31. Laniado ME, Ockrim S, Marronaro A, Tubaro A, Roco B, Carter S. Bladder wall thickness and free flow rate in a logistic regresion predict patients who do not have lower urinary tract obstruction on presure/flow urodynamic studies. J Urol 2002;167(Suppl 4) [Abstract 1065]

32. International Continence Society. First report on the standardisation of terminology of lower urinary tract function. Br J Urol 1976;48(1):39-42.

33. Susset J. Relationship between clinical urodynamics and pathologic findings in prostatic obstruction. In: Himman F, ed. Benign prostatic hypertrophy. New York: Springer-Verlag, 1983.

34. Salinas Casado J, Sánchez Blasco E, Vírseda Chamorro M, Bravo de Rueda C, Rapariz González M, Esteban Fuertes M, Resel Estévez L. Fiabilidad de la estimación del volumen vesical postmiccional mediante ultrasonidos. Comparación de dos métodos ecográficos. Arch Esp Urol 1996;49(1):35-40.

35. Ball AJ, Feneley RC, Abrams PH. The natural history of untreated «prostatism». Br J Urol 1981;53(6):613-6.

36. Van Mastrigt R, Rollema HJ. The prognostic value of bladder contractility in transurethral resection of the prostate. J Urol. 1992 Dec;148(6):1856-60.

37. Thomas AW, Cannon A, Bartlett E, Ellis-Jones J, Abrams P. The natural history of lower urinary tract dysfunction in men: minimum 10-year urodynamic follow-up of untreated bladder outlet obstruction. BJU Int. 2005 Dec;96(9):1301-6.

38. Abrams P, Griffiths DJ. The assessment of prostatic obstruction from urodynamic measurements and from residual urine. Br J Urol 1979;51:12934.

*39. Oelke M, Mamoulakis C, Ubbink DT, de la Rosette JJ, Wijkstra H. Manual versus automatic bladder wall thickness measurements: a method comparison study. World J Urol. 2009 Feb 24. [Epub ahead of print]

*40. Kojima M, Inui E, Ochiai A, Naya Y, Ukimura $\mathrm{O}$, Watanabe $\mathrm{H}$. Ultrasonic estimation of bladder weight as a measure of bladder hypertrophy in men with infravesical obstruction: a preliminary report. Urology 1996;47(6):942-7.

41. Kojima M, Inui E, Ochiai A, Naya Y, Ukimura O, Watanabe H. Noninvasive quantitative estimation of infravesical obstruction using ultrasonic measurement of bladder weight. J Urol 1997; 157(2):476-9. 
42. Canepa G, Capponi G, Campodonico F, Maffezzini M. The influence of TUR or open prostatectomy on ultrasound estimated bladder weight (UEBW): a prospective study on 26 patients. Neurourol Urodyn 2003;22(5):424-5.

43. Miyashita H, Kojima M, Miki T. Ultrasonic measurement of bladder weight as a possible predictor of acute urinary retention in men with lower urinary tract symptoms suggestive of benign prostatic hyperplasia. Ultrasound Med Biol. 2002 Aug;28(8):985-90.

44. Ochiai A, Kojima M. Correlation of ultrasoundestimated bladder weight with ultrasound appearance of the prostate and postvoid residual urine in men with lower urinary tract symptoms.Urology. 1998 May;51(5):722-9.

45. Ukimura O, Kojima M, Iwata T, Inaba M, Miki T. Ultrasonic measurement of bladder weight as a novel urodynamic modality. Adv Exp Med Biol. 2003;539(Pt A):311-5.

46. Elbadawi A, Yalla SV, Resnick NM. Structural basis of geriatric voiding dysfunction. J Urol 1993;150:1650-5, 1656-67, 1668-80, 1681-95.

47. Bates P, Bradley WE, Glen E, Griffiths D, Melchior H, Rowan D, Sterling A, Zinner N, Hald T. The standardization of terminology of lower urinary tract function. J Urol 1979;121(5):551-4.

48. Yamamoto T, Sakakibara R, Uchiyama T, Liu Z, Ito T, Awa Y, Yamanishi T, Hattori T. Neurological diseases that cause detrusor hyperactivity with impaired contractile function. Neurourol Urodyn 2006; 25(4):356-60.

49. Blaivas JG. Discussion: a framework for diagnosis of idiopathic overactive bladder. Urology 1997;50(Suppl 6A):34.

50. Turner-Warwick R, Whiteside CG, Worth PH, Milroy EJ, Bates CP. A urodynamic view of the clinical problems associated with bladder neck dysfunction and its treatment by endoscopic incision and trans-trigonal posterior prostatectomy. Br J Urol 1973; 45(1):44-59.

51. Salinas Casado J, Prieto Chaparro L, Martín Garcia C, Rapariz González M, Virseda Chamorro M, Nacarino Corbacho L, Resel Estévez L Utilidad de la aceleración del flujo miccional en el diagnóstico de la inestabilidad vesical. Arch Esp Urol
1993;46(10):891-5.

52. Jones KW, Schoenberg HW. Obstruction and the uninhibitable detrusor. In: Himman F, ed. Benign prostatic hypertrophy. New York: Springer-Verlag, 1983; chap 58, pp. 707-10.

53. Speakman MJ, Sethia KK, Fellows GJ, Smith JC. A study of the pathogenesis, urodynamic assessment and outcome of detrusor instability associated with bladder outflow obstruction. Br J Urol 1987;59(1):40-4.

**54. Hyman MJ, Groutz A, Blaivas JG. J Urol. 2001 Aug;166(2):550-2; discussion 553. Detrusor instability in men: correlation of lower urinary tract symptoms with urodynamic findings.

55. Stephenson TP. The interpretation of conventional urodynamics. In Mundy, Stephenson, Wein, eds. Urodynamics: principles, paractice and application. Edinburgh: Churchill Livingstone, 1994.

56. Salinas J, Vírseda M, Fuente MP, Mellado F, Uson AC. A study of the viscoelastic properties in dogs. Urol Int 1992;49:185-90.

57. McGuire EJ. The role of urodynamic investigation in the assessment of benign prostatic hypertrophy. J Urol 1992;148(4):1133-6.

58. Webb RJ, Styles RA, Griffiths CJ, Ramsden PD, Neal DE. Ambulatory monitoring of bladder pressures in patients with low compliance as a result of neurogenic bladder dysfunction. $\mathrm{Br} \mathrm{J}$ Urol 1989;64(2):150-4.

**59. Robinson D, Anders K, Cardozo L, Dixon A. Bladder wall thickness and the over-active bladder: do contractions count? Neurourol Urodyn 2003;22(5):422-3.

60. Minardi D, Piloni V, Amadi A, El Asmar Z, Milanese G, Muzzonigro G. Correlation between urodynamics and perineal ultrasound in female patients with urinary incontinence. Neurourol Urodyn 2007; 26(2): 176-82.

*61. Khullar V, Cardozo LD, Salvatore S, Hill S. Ultrasound: a noninvasive screening test for detrusor instability. Br J Obstet Gynaecol 1996 Sep;103(9):904-8.

**62. Lekskulchai O, Dietz HP. Ultrasound Obstet Gynecol. 2008 Sep;32(4):535-9.

Detrusor wall thickness as a test for detrusor overactivity in women. 